\title{
Seasonal change of total sleep time and open air temperature
}

\author{
Tomoyuki Kawada
}

Received: 11 March 2010 /Revised: 16 March 2010 / Accepted: 19 March 2010 /Published online: 6 April 2010

(C) ISB 2010

A recent paper by Okamoto-Mizuno and Tsuzuki (2009) reported the seasonal variation of sleep in the elderly over 61 years old in relation to skin temperature. They monitored sleep by using a wrist accelerometer system for five consecutive days. The author has an experience of long-term monitoring of total sleep time for one person by using the apparatus of the same mechanism and validated that total sleep time is disturbed in summer compared with other seasons. The author also analyzed total sleep time in connection with open air temperature.

The age of the subject is 50 years old in 2010. Measurements were conducted for 5 years continuously since November 2002. Sleep was monitored consecutively using a piezo-electric accelerometer, named Actiwatch ${ }^{\circledR}$ (Mini Mitter, Sunriver), on the wrist of their non-dominant arm, removing it only during periods when it could get wet. The sampling frequency was set at $32 \mathrm{~Hz}$ and the sensitivity at $0.05 \mathrm{G}$, using $1 \mathrm{~min}$ as the unit, and the number of body movements was calculated by the summation of active electricity counts. If the count is 40 or more, we judged it as wake. The count of 40 is adopted as the cut-off value to differentiate sleep and wake (Benson et al. 2004). When under 40, the number of two epochs before the target epoch $\times 0.04+$ number of one epoch before the target epoch $\times 0.2+$ number of the target epoch + number of one epoch after the target epoch $\times 0.2+$ number of two epochs after the target epoch $\times 0.04$ exceeds 40 , was judged as wake. The author checked the validity of this activity-monitoring device in healthy subjects. Using this algorithm, the agreement between Actiwatch ${ }^{\circledR}$ and the sleep polygraph was $88.4 \%$ on average, based on 24 nights, and Pearson's correlation coefficient of total sleep time (TST)

T. Kawada $(\bowtie)$

Nippon Medical School,

Bunkyo, Tokyo, Japan

e-mail: kawada@nms.ac.jp between Actiwatch $^{\circledR}$ and the sleep polygraph was 0.85 (Kawada et al. 2002). The author gathered information of monthly average of open air temperaturefrom the local weather station. Four seasons; spring, summer, fall, and winter; were defined in this study as December-February, March-May, June-August, and September-November, respectively. Before calculating seasonal means and standard deviations of total sleep time, monthly average values of total sleep time was primarily calculated. So the number of each sample in spring, summer, fall, and winter was 15,15 , 14 , and 15 , respectively.

The means (and standard deviations in parentheses) of total sleep time in spring, summer, fall, and winter were 364.5 (14.2), 351.4 (16.0), 365.2 (13.0), and 366.5 minutes (15.3). The mean value of total sleep time in summer was significantly lower that that in winter by Tukey's multiple comparison. Furthermore, Pearson's moment correlation coefficient between mean open air temperature and the mean value of total sleep time in month was significant $(r=-0.386$, $n=59, p=0.003$ ).

From long-term monitoring of total sleep time by accelerometer, sleep is disturbed in summer compared with winter, partly explained by the open air temperature.

\section{References}

Benson K, Friedman L, Noda A, Wicks D, Wakabayashi E, Yesavage J (2004) The measurement of sleep by actigraphy: direct comparison of 2 commercially available actigraphs in a nonclinical population. Sleep 27:986-989

Kawada T, Kuroiwa M, Sasazwa Y, Suzuki S, Tamura Y (2002) Sleep time monitoring by accelerometer in two subjects for one year. J Sound Vibration 250:75-82

Okamoto-Mizuno K, Tsuzuki K (2009) Effects of season on sleep and skin temperature in the elderly. Int J Biometeorol Dec 30. [Epub ahead of print] 